\title{
Disfunção sexual em mulheres com câncer do colo do útero submetidas à radioterapia: análise de conceito
}

\author{
Sexual dysfunction in women with cervical cancer undergoing radiotherapy: concept analysis \\ Disfunción sexual en mujeres con cáncer de cuello uterino sometidas a radioterapia: análisis de \\ concepto
}

\begin{abstract}
Thaynan Gonçalves da Silva ${ }^{1}$ (1) Kilders Mitshel Lucas de Oliveira ${ }^{1}$ (B) Sheila Coelho Ramalho Vasconcelos Morais ${ }^{1}$ (1) Jaqueline Galdino Albuquerque Perrelli ${ }^{1}$ (B) Santana de Maria Alves de Sousa² (1) Francisca Márcia Pereira Linhares ${ }^{1}$ (1)
\end{abstract}

1. Universidade Federal de Pernambuco. Recife, PE, Brasil.

2. Universidade Federal do Maranhão. São Luís, MA, Brasil.
Autor correspondente:

Thaynan Gonçalves da Silva.

E-mail: t.thaygoncalves@gmail.com.

Recebido em 09/10/2020.

Aprovado em 28/04/2021.

\section{Resumo}

Objetivo: analisar o conceito de disfunção sexual em mulheres com câncer do colo do útero submetidas a tratamento radioterápico. Método: estudo de análise de conceito fundamentado no referencial metodológico proposto por Walker e Avant, o qual é composto por oito etapas: seleção do conceito; determinação dos objetivos da análise conceitual; identificação dos possíveis usos do conceito; determinação dos atributos críticos ou essenciais; construção de um caso modelo; construção de casos adicionais; identificação dos antecedentes e consequentes do conceito; e definição das referências empíricas. Resultados: a amostra final resultou em 11 artigos, que identificaram 4 atributos críticos, 18 antecedentes e 07 consequentes do conceito, os quais permitiram a construção de uma definição conceitual e elaboração de um caso-modelo, com a finalidade de exemplificar o contexto social no qual o conceito encontra-se inserido. Conclusão e implicações para prática: alterações na estrutura corporal, déficit de conhecimento, diminuição da frequência das relações sexuais, dispareunia e indiferença do parceiro são alguns dos principais antecedentes e consequentes da disfunção sexual na população estudada. Entretanto, esses fatores ainda são pouco conhecidos, o que limita a realização de açães de promoção, prevenção e reabilitação sexual, sobretudo no campo da enfermagem.

Palavras-chave: Diagnóstico de enfermagem; Radioterapia; Neoplasias do colo do útero; Sexualidade; Saúde da Mulher.

\section{Abstract}

Objective: to analyze the concept of sexual dysfunction in women with cervical cancer undergoing radiotherapy. Method: a concept analysis study based on the methodological framework proposed by Walker and Avant, which consists of eight stages: concept selection; determination of the objectives of the conceptual analysis; identification of possible uses of the concept; determination of critical or essential attributes; construction of a model case; construction of additional cases; identification of the antecedents and consequences of the concept; and definition of empirical references. Results: the final sample resulted in 11 articles, which identified 4 critical attributes, 18 antecedents and 07 consequences of the concept, which allowed the construction of a conceptual definition and the elaboration of a model case in order to exemplify the social context in which the concept is inserted. Conclusion and implications for practice: changes in body structure, lack of knowledge, decreased frequency of sexual intercourse, dyspareunia, and partner's indifference are some of the main antecedents and consequences of sexual dysfunction in the studied population. However, these factors are still little known, which limits the performance of actions of promotion, prevention and sexual rehabilitation, especially in the field of nursing.

Keywords: Nursing diagnosis; Radiotherapy; Uterine cervical neoplasms; Sexuality; Women's Health.

\section{REsumen}

Objetivo: analizar el concepto de disfunción sexual en mujeres con cáncer de cuello uterino sometidas a tratamiento radioterápico. Método: estudio de análisis de concepto basado en el marco metodológico de Walker y Avant, compuesto por ocho etapas: selección del concepto; determinación de los objetivos del análisis conceptual; identificación de posibles usos del concepto; determinación de atributos críticos o esenciales; construcción de un caso modelo; construcción de casos adicionales; identificación de antecedentes y consecuentes del concepto; y definición de referencias empíricas. Resultados: La muestra final resultó en 11 artículos: 04 críticos, 18 antecedentes y 07 consecuentes del concepto, lo que permitió la construcción de una definición conceptual y la elaboración de un caso modelo con el fin de ejemplificar el contexto social en que se inserta el concepto. Conclusión e implicaciones para la práctica: Cambios en la estructura corporal, déficit de conocimiento, disminución de la frecuencia de las relaciones sexuales, dispareunia y la indiferencia de la pareja son algunos de los principales antecedentes y consecuentes de la disfunción sexual en la población estudiada. Sin embargo, estos factores aún son poco conocidos, lo que limita la realización de acciones de promoción, prevención y rehabilitación sexual, especialmente en el campo de la enfermería.

Palabras clave: Diagnóstico de enfermería; Radioterapia; Neoplasias del cuello uterino; Sexualidad; Salud de la Mujer. 


\section{INTRODUÇÃO}

O câncer do colo do útero é responsável por 265 mil óbitos, a cada ano, em todo o mundo, e ocupa a quarta causa de morte por câncer na população feminina. No Brasil, estimamse 16.590 casos novos para cada ano do triênio 2020-2022. No Nordeste brasileiro, esse tipo de câncer está entre as neoplasias femininas mais frequentes, e apresenta altas taxas de incidência e mortalidade em países em desenvolvimento, devido à estrutura deficiente dos programas de rastreamento ${ }^{1-4}$.

A etiologia é multifatorial, e está associada principalmente à baixa condição socioeconômica e à infecção persistente pelo papilomavírus humano (HPV) ${ }^{2-4}$. Em estágios iniciais, o tratamento de primeira linha é a ressecção cirúrgica. Nos casos mais avançados, opta-se por quimioterapia e/ou radioterapia 4 . O tratamento radioterápico ocasiona alterações significantes no epitélio vaginal, com mudanças expressivas na qualidade de vida e funcionamento sexual ${ }^{5,6}$. Dentre elas, citam-se: dispareunia, diminuição na sensibilidade e elasticidade vaginal, além da redução na capacidade de atingir o orgasmo ${ }^{5}$.

Essas alterações levam à diminuição da função sexual, uma vez que a sexualidade feminina é composta por uma interação de fatores físicos, sociais, comportamentais e culturais. $O$ desequilíbrio entre esses fatores pode acarretar danos significativos ao prazer sexual e levar à disfunção sexual ${ }^{5,7}$. Esses danos afetam negativamente a qualidade de vida em decorrência da presença de algum grau de disfunção sexual, após o tratamento para as neoplasias cervicais, sendo necessária a realização de aconselhamento e acompanhamento por profissionais da saúde - médicos, enfermeiros e psicólogos -, antes e após o tratamento, a fim de que suas necessidades em relação às questões sexuais sejam avaliadas e atendidas ${ }^{1}$.

Entretanto, apesar da alta prevalência da disfunção sexual nessa fase de pós-tratamento, ainda é pouco estudada, principalmente como um Diagnóstico de Enfermagem (DE), tendo em vista que essa população é assistida de forma direta, por enfermeiros, nos serviços especializados de tratamento oncológico. Nesse sentido, torna-se importante compreender a complexidade do comportamento sexual de mulheres submetidas a tratamento radioterápico, pois o contexto as induz a vivenciarem a diminuição do desejo sexual. Além disso, percebe-se que os profissionais de saúde - especialmente médicos e enfermeiros - têm dificuldade em abordar essa temática, pela falta de conhecimento, crenças pessoais, ou ainda pelo fato de estarem mais focados no tratamento da doença ${ }^{8,9}$.

Desse modo, identificar os atributos críticos, antecedentes e consequentes da disfunção sexual, nessa população, torna-se relevante para a prática assistencial, uma vez que o conhecimento desses componentes permite, ao enfermeiro, elaborar DE voltados para a sexualidade feminina, planejar e implementar ações de promoção da saúde para a qualidade de vida dessas mulheres, a fim de que possam vivenciar a sexualidade de forma mais prazerosa e satisfatória ${ }^{10,11}$.

Além disso, a identificação de novos componentes diagnósticos possibilita ao enfermeiro maior compreensão e clareza do conceito estudado, o que subsidia e qualifica sua prática nos serviços especializados de tratamento oncológico, referente às ações de educação em saúde e prevenção de distúrbios na sexualidade feminina.

Assim, considerando a relevância da temática em questão, este estudo tem o objetivo de analisar o conceito de disfunção sexual em mulheres com câncer do colo do útero, submetidas a tratamento radioterápico.

\section{MÉTODO}

Trata-se de análise de conceito, cujo objetivo é realizar avaliação e descrição cuidadosa de um termo e seus usos ${ }^{12}$. Esse conceito não se restringe a uma palavra, mas representa a ideia que é expressa ao utilizá-la ${ }^{13}$. Neste estudo, analisou-se o conceito de Disfunção Sexual em mulheres com câncer do colo do útero, submetidas a tratamento radioterápico, segundo o referencial teórico-metodológico proposto por Walker e Avant ${ }^{12}$, o qual é constituído por oito etapas.

Na primeira etapa, - escolha do conceito -, foi definido que o conceito a ser analisado seria disfunção sexual. Optouse em incluir o prefixo "dis" (julgamento), para especificar o que deveria ser investigado. Na segunda etapa, definiram-se os objetivos da análise de conceito, a saber: determinar os atributos críticos, antecedentes e os consequentes da disfunção sexual em mulheres com câncer do colo do útero, submetidas a tratamento radioterápico; elaborar a definição conceitual de disfunção sexual; e definir as referências empíricas. A terceira etapa - identificação dos usos do conceito - foi realizada por meio de uma revisão integrativa de literatura, que seguiu as etapas propostas por Whittemore e Knafl ${ }^{14}$.

Para a elaboração da pergunta de pesquisa da revisão integrativa - Quais as evidências científicas acerca dos atributos críticos, antecedentes e dos consequentes da disfunção sexual em mulheres com câncer do colo do útero submetidas a tratamento radioterápico? -, utilizou-se a estratégia $\mathrm{PICo}^{15}$, onde $\mathrm{P}=$ População (mulheres), l= Fenômeno (disfunção sexual), Co= Contexto (câncer do colo do útero e tratamento radioterápico).

A busca na literatura foi realizada por meio eletrônico, via portal de periódicos CAPES, nas bases de dados CINAHL, Medline, PubMed, Scopus e Web of Science. Procedeu-se à busca de forma não controlada, por meio de descritores indexados no MeSH (Medical Subject Headings) e DeCS (Descritores em Ciências da Saúde), nos idiomas português, inglês ou espanhol. Os descritores utilizados foram: sexual dysfunction, radiotherapy, toxicity, pelvic neoplasms e women. Empregaram-se os seguintes cruzamentos: radiotherapy AND "sexual dysfunction" AND women; Radiotherapy AND "sexual dysfunction" AND "pelvic neoplasms"; radiotherapy AND "pelvic neoplasms" AND toxicity. Utilizou-se o operador boleano "AND" para o cruzamento dos referidos descritores. Os cruzamentos foram realizados de forma semelhante em todas as bases de dados, com a finalidade de identificar o maior número possível de artigos.

A busca foi realizada por dois investigadores independentes, durante todo o processo, segundo recomendações de duplo 
cegamento. Em caso de divergências entre os pesquisadores, estas foram resolvidas por meio de um terceiro investigador, que emitiu o parecer final. Foram incluídos artigos originais que respondessem à questão da pesquisa, estudos disponíveis na íntegra nas bases de dados selecionadas, nos idiomas português, inglês ou espanhol, publicados no período de 2015 a 2019. O recorte temporal foi utilizado para atender à recomendação da Taxonomia proposta pela North American Nursing Diagnosis Association (NANDA-I), ${ }^{16}$ que sugere a utilização de publicações dos últimos cinco anos para realização de estudos de revisão e validação de diagnósticos de enfermagem.

Os estudos identificados foram submetidos à leitura de título e resumo, de acordo com os critérios de elegibilidade previamente estabelecidos. Em caso de dúvidas, seguiu-se realizando a leitura na íntegra. Para coleta dos dados, foi utilizado um instrumento adaptado de Mangueira ${ }^{17}$, previamente elaborado, de modo que os dados pudessem ser extraídos em sua totalidade e o risco de erros na transcrição fosse minimizado. O rigor metodológico foi avaliado por meio do Critical Appraisal Skills Programm (CASP) ${ }^{18}$, específico para cada tipo de estudo. Receberam pontuação $A$ (6 a 10 pontos) os estudos com boa qualidade metodológica e viés reduzido; e pontuação $B$ (até 5 pontos), os estudos com qualidade metodológica satisfatória e risco de viés.

O nível de evidência dos estudos selecionados foi avaliado por meio da classificação proposta por Melnyk e Fineout-Overholt ${ }^{19}$, a qual classifica os estudos do nível I (evidências oriundas de revisão sistemática ou metanálise) até o nível VII (evidências oriundas de opinião de autoridades e/ou relatório de comitês de especialistas).

Mediante a busca inicial nas bases de dados e portais eletrônicos, foram encontradas 3.886 publicações, das quais selecionaram-se 739 para leitura do título e resumo, após aplicação dos critérios de elegibilidade. Destas, excluíram-se 674 por não responderem à pergunta do estudo, e 17 por estarem duplicadas. Desse modo, 48 publicações foram selecionadas para leitura na íntegra; após leitura completa, excluíram-se 37 publicações, porque não respondiam à questão da pesquisa. A amostra final resultou 11 artigos, dos quais foram extraídos os atributos críticos, antecedentes e consequentes da disfunção sexual em mulheres com diagnóstico de câncer do colo do útero, submetidas a tratamento radioterápico (Figura 1).

Com relação à caracterização dos artigos, a maioria foi obtida na base de dados Scopus (45,5\%), estava no idioma inglês (100\%), e países do continente asiático foram os principais locais de publicação (54,6\%). Observou-se linearidade quanto ao número de publicações realizadas nos últimos cinco anos acerca do objeto de estudo; entretanto, as publicações concentraramse na área médica $(90,9 \%)$, sendo apenas uma publicação de enfermagem $(9,1 \%)$, a qual não abordava o diagnóstico de enfermagem disfunção sexual para população estudada.

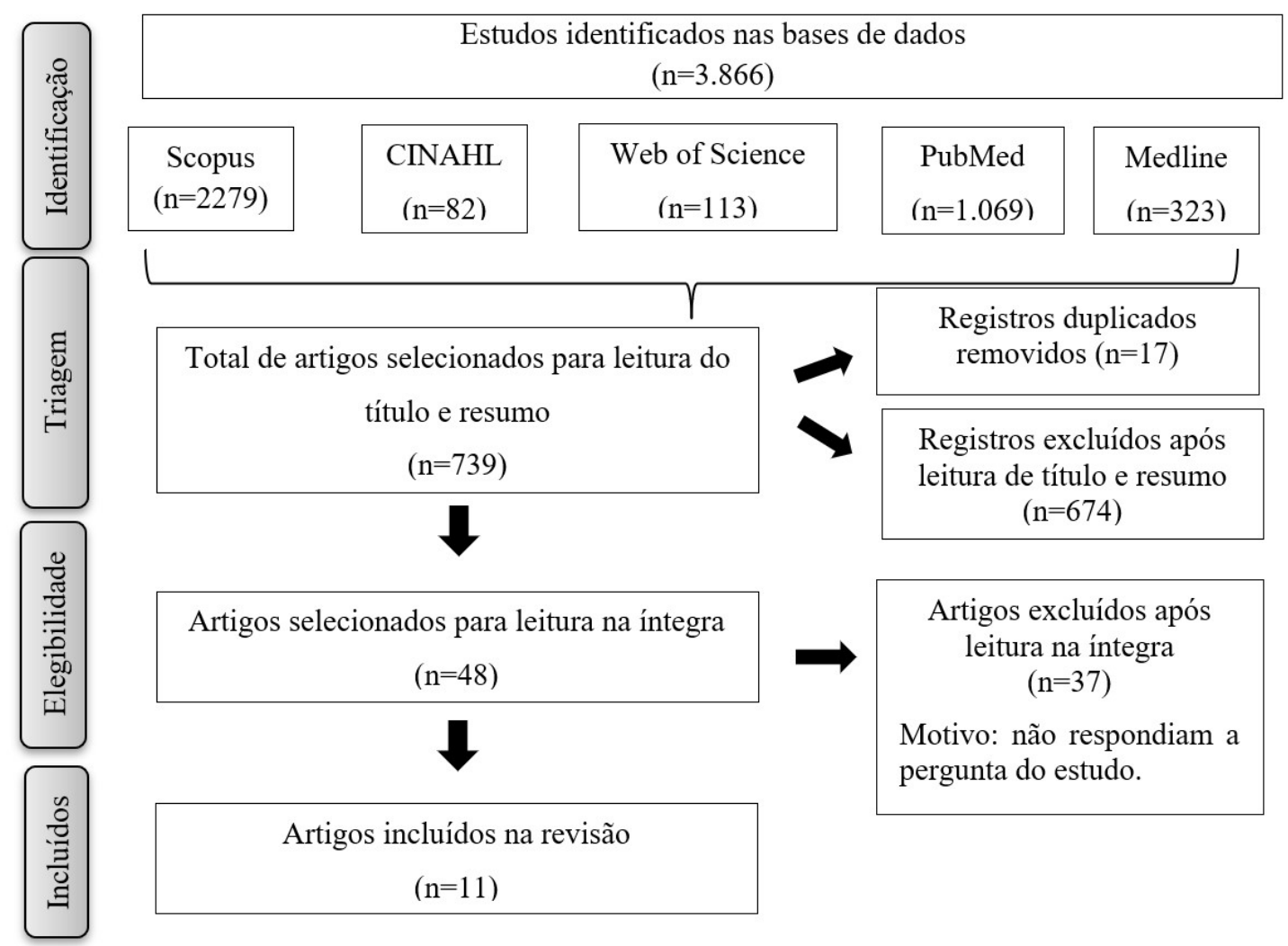

Figura 1. Fluxograma da revisão integrativa. Recife, PE, Brasil, 2020. Fonte: Adaptado de Ferrari ${ }^{20}$ 
Quanto ao nível de evidência dos 11 artigos selecionados, a maioria $(63,7 \%)$ foi classificada como estudos descritivos e obtiveram nívelVI. Ao avaliar o rigor metodológico, 90,9\% dos artigos obtiveram pontuação maior que seis e foram classificados com nível $A$.

Na quarta etapa da análise de conceito, determinaram-se os atributos críticos ou essenciais, a partir da literatura estudada. Nessa etapa, foram identificadas palavras ou expressões que apareceram continuamente na literatura e caracterizaram a essência do conceito. Em momento posterior, quinta etapa, construiu-se um caso-modelo com a finalidade de exemplificar o uso do conceito, o qual demonstra todos os seus atributos críticos encontrados na revisão de literatura ${ }^{12}$. Neste estudo, o caso-modelo foi construído a partir da literatura estudada, e da vivência profissional das autoras com o fenômeno em estudo.

A sexta etapa caracteriza-se pela identificação de casos adicionais e deve ser realizada apenas se o conceito não estiver claro no caso modelo ${ }^{12}$. Neste estudo, a construção de casos adicionais foi desnecessária. Na sétima etapa, foram identificados os antecedentes (eventos necessários para ocorrência do fenômeno) e consequentes (situações decorrentes da presença do conceito), a partir da revisão integrativa de literatura realizada ${ }^{12}$.

Na última etapa da análise do conceito, determinaram-se os referenciais empíricos, que correspondem aos fenômenos observáveis na prática, e quando presentes demonstram a ocorrência do conceito em $\mathrm{si}^{12}$. Por meio de uma busca ampliada em livros, artigos, dissertações, teses e manuais, foram elaboradas as definições conceituais e operacionais dos antecedentes e consequentes do conceito em estudo.

\section{RESULTADOS}

Na análise dos artigos, foi possível observar que o conceito de disfunção sexual é utilizado em todos os ambientes em que ocorre o cuidado em mulheres com câncer do colo do útero, submetidas a tratamento radioterápico. Identificaramse 4 atributos críticos, 18 antecedentes e 7 consequentes da disfunção sexual em mulheres com diagnóstico de câncer do colo do útero, submetidas a tratamento radioterápico. Dentre esses, apenas três antecedentes encontram-se citados na última versão da Taxonomia NANDA-I ${ }^{16}$, sendo eles: diminuição da libido, atividades de educação em saúde deficiente e estenose vaginal. Em relação aos consequentes, dos sete componentes identificados, apenas insatisfação satisfação sexual encontra-se citada na referida Taxonomia (Quadro 1). Diante desse contexto, a inclusão de novos antecedentes e consequentes da disfunção sexual na Taxonomia NANDA-I torna-se necessária e oportuna.

A partir dos atributos identificados (Quadro 1), elaborou-se a seguinte definição conceitual para o diagnóstico de enfermagem Disfunção Sexual: alteração na resposta sexual saudável em decorrência de mudança na estrutura anatômica e/ou fisiológica corporal, a qual pode ser influenciada por fatores psicológicos, sociais e culturais.

Com a finalidade de exemplificar o contexto social no qual o conceito encontra-se inserido, elaborou-se o seguinte caso-modelo: M.R.S., 45 anos, sexo feminino, comparece a consulta de revisão após finalizar as sessões de radioterapia para o tratamento de um câncer do colo de útero diagnosticado recentemente. Durante a consulta, ela informou dificuldade para manter relações sexuais com seu companheiro, uma vez que sentia muita dor durante 0 coito e "secura" vaginal, além do medo de recidiva da doença, o que ocasionou diminuição do seu desejo sexual. Ao avaliar os exames laboratoriais de M.R.S., o profissional observou diminuição dos níveis de estradiol e progesterona. Durante a realização do exame físico, também foi identificada diminuição da elasticidade e do comprimento vaginal. A partir dos achados durante a realização da anamnese e exame físico, o profissional orientou M.R.S. quanto aos exercícios vaginais que podiam ser realizados para promover dilatação do canal vaginal, assim como o uso de lubrificantes vaginais para facilitar o coito. O profissional também explicou que a relação sexual não era fator causador de uma possível recidiva, e a encaminhou ao Serviço de Psicologia, para que houvesse atendimento integral de suas necessidades biopsicossociais ${ }^{1,5,21,22,24,27}$.

\section{DISCUSSÃO}

Os dados deste estudo revelam prevalência de pesquisas quanto à disfunção sexual feminina em mulheres com diagnóstico de neoplasia do colo do útero, submetidas a tratamento radioterápico no continente asiático, o que pode ser explicado pela elevada taxa de disfunção sexual feminina no referido continente, com variação entre $46 \%$ e $73 \%{ }^{30}$. Na América-Latina, essa taxa varia entre $25 \%$ e $43 \%$; entretanto, não foram encontrados estudos dessa natureza ${ }^{31,32}$.

Para a Organização Mundial da Saúde ${ }^{32}$, a disfunção sexual feminina tornou-se importante problema de saúde pública, uma vez que interfere diretamente na qualidade de vida. Não obstante, a abordagem deste assunto, nos atendimentos realizados por profissionais de saúde - principalmente por enfermeiros -, ainda é incipiente, o que contribui para a cronicidade do problema ${ }^{5}$.

Dentre os fatores relacionados mais frequentes, têm-se a comunicação deficiente entre profissional de saúde-paciente e o número reduzido de atividades de educação em saúde nos serviços de oncologia. A ocorrência desses eventos pode ser explicada pela dificuldade dos profissionais em abordar o tema durante as consultas de rotina, seja pela falta de conhecimento suficiente e crenças pessoais, ou ainda pelo foco na doença de base. Outro fator contribuinte para essa problemática é a vergonha que muitas mulheres sentem em abordar o tema durante as consultas, o que as impede de buscar ajuda profissional $\left.\right|^{5,33}$.

O desenvolvimento de ações educativas em saúde e uma boa comunicação podem reduzir as disfunções sexuais mediante o esclarecimento de dúvidas, medos e anseios. Além disso, a realização de tais ações permite uma construção conjunta de conhecimentos, troca de experiências e reforça a confiança entre profissional e paciente ${ }^{34}$.

A análise de conceito também revelou que angústia, ansiedade, vergonha e medo são fatores relacionados que contribuem para a disfunção sexual em mulheres com diagnóstico de neoplasia do colo do útero, submetidas a tratamento radioterápico. Durante ou 
Quadro 1. Caracterização dos artigos incluídos na Revisão Integrativa, segundo título, ano, atributo, antecedentes e consequentes da disfunção sexual em mulheres com diagnóstico de câncer do colo do útero, submetidas a tratamento radioterápico. Recife, PE, Brasil, 2020.

\begin{tabular}{|c|c|c|c|c|}
\hline Título & Ano & Atributos & Antecedentes & Consequentes \\
\hline $\begin{array}{l}\text { Sexual function } \\
\text { in South African } \\
\text { women treated for } \\
\text { cervical cancer }\end{array}$ & 2019 & $\begin{array}{l}\text { Alteração na estrutura } \\
\text { do epitélio vaginal; } \\
\text { diminuição dos níveis de } \\
\text { estradiol e progesterona; } \\
\text { influência de fatores } \\
\text { psicológicos, sociais e } \\
\text { culturais; alteração em } \\
\text { uma das fases da resposta } \\
\text { sexual saudável (desejo, } \\
\text { excitação, orgasmo e } \\
\text { resolução). }\end{array}$ & $\begin{array}{l}\text { Fatores relacionados: } \\
\text { medo; diminuição da } \\
\text { libido; diminuição da } \\
\text { lubrificação vaginal; } \\
\text { estenose vaginal, } \\
\text { sangramento vaginal } \\
\text { anormal. } \\
\text { Condições associadas: } \\
\text { radioterapia; baixa } \\
\text { escolaridade. } \\
\text { População em risco: } \\
\text { mulheres com idade igual } \\
\text { ou maior de } 40 \text { anos. }\end{array}$ & $\begin{array}{l}\text { Características } \\
\text { definidoras: diminuição } \\
\text { da frequência das } \\
\text { relações sexuais; } \\
\text { dispareunia. }\end{array}$ \\
\hline $\begin{array}{l}\text { Sexual experience } \\
\text { of women after } \\
\text { pelvic radiotherapy } \\
\text { for cervical cancer }\end{array}$ & 2016 & $\begin{array}{l}\text { Alteração na estrutura } \\
\text { do epitélio vaginal; } \\
\text { diminuição dos níveis de } \\
\text { estradiol e progesterona; } \\
\text { influência de fatores } \\
\text { psicológicos, sociais e } \\
\text { culturais. }\end{array}$ & $\begin{array}{l}\text { Fatores relacionados: } \\
\text { comunicação deficiente } \\
\text { entre profissional- } \\
\text { paciente; atividades } \\
\text { de educação em saúde } \\
\text { deficiente; ansiedade, } \\
\text { distúrbio de autoimagem; } \\
\text { ausência de parceiro } \\
\text { fixo; diminuição da } \\
\text { libido, estenose vaginal; } \\
\text { sangramento vaginal } \\
\text { anormal. } \\
\text { Condições associadas: } \\
\text { radioterapia, depressão, } \\
\text { baixa escolaridade. } \\
\text { População em risco: } \\
\text { mulheres com idade igual } \\
\text { ou maior de } 40 \text { anos. }\end{array}$ & $\begin{array}{c}\text { Características } \\
\text { definidoras: indiferença } \\
\text { do parceiro; sentimento } \\
\text { de obrigatoriedade em } \\
\text { manter relação sexual; } \\
\text { dispareunia. }\end{array}$ \\
\hline $\begin{array}{l}\text { Autologous } \\
\text { platelet-released } \\
\text { growth factor and } \\
\text { sexual dysfunction } \\
\text { amendment: a } \\
\text { pilot clinical trial } \\
\text { of successful } \\
\text { improvement } \\
\text { sexual dysfunction } \\
\text { after pelvic } \\
\text { radiotherapy }\end{array}$ & 2019 & $\begin{array}{l}\text { Alteração na estrutura do } \\
\text { epitélio vaginal. }\end{array}$ & $\begin{array}{l}\text { Fatores relacionados: } \\
\text { diminuição da lubrificação } \\
\text { vaginal; estenose vaginal. }\end{array}$ & $\begin{array}{l}\text { Características } \\
\text { definidoras: insatisfação } \\
\text { sexual; diminuição da } \\
\text { frequência das relações } \\
\text { sexuais; dispareunia. }\end{array}$ \\
\hline $\begin{array}{l}\text { Radiotherapy for } \\
\text { cervical cancer- } \\
\text { impact on the } \\
\text { vaginal epithelium } \\
\text { and sexual } \\
\text { function }\end{array}$ & 2017 & $\begin{array}{l}\text { Alteração na estrutura do } \\
\text { epitélio vaginal. }\end{array}$ & $\begin{array}{l}\text { Fatores relacionados: } \\
\text { diminuição da lubrificação } \\
\text { vaginal; estenose vaginal; } \\
\text { edema vaginal. } \\
\text { Condições associadas: } \\
\text { radioterapia. }\end{array}$ & $\begin{array}{c}\text { Características } \\
\text { definidoras: dispareunia. }\end{array}$ \\
\hline
\end{tabular}

Fonte: Elaborada pelos autores, Recife, PE, Brasil, 2020 
Quadro 1. Continuação...

\begin{tabular}{|c|c|c|c|c|}
\hline Título & Ano & Atributos & Antecedentes & Consequentes \\
\hline $\begin{array}{l}\text { Survey of cervical } \\
\text { cancer survivors } \\
\text { regarding quality } \\
\text { of life and sexual } \\
\text { function }{ }^{23}\end{array}$ & 2016 & & $\begin{array}{l}\text { Fatores relacionados: } \\
\text { comunicação deficiente } \\
\text { entre profissional- } \\
\text { paciente; atividades } \\
\text { de educação em saúde } \\
\text { deficiente; medo; } \\
\text { ansiedade; diminuição da } \\
\text { libido; estenose vaginal. } \\
\text { Condições associadas: } \\
\text { radioterapia; cirurgia; } \\
\text { depressão; baixa } \\
\text { escolaridade; ocupação } \\
\text { informal. } \\
\text { População em risco: } \\
\text { mulheres com idade igual } \\
\text { ou maior de } 40 \text { anos. }\end{array}$ & $\begin{array}{c}\text { Características } \\
\text { definidoras: insatisfação } \\
\text { sexual; dispareunia. }\end{array}$ \\
\hline $\begin{array}{l}\text { Pelvic radiotherapy } \\
\text { and sexual function } \\
\text { in women }\end{array}$ & 2015 & $\begin{array}{l}\text { Alteração na estrutura do } \\
\text { epitélio vaginal; alteração } \\
\text { em uma das fases da } \\
\text { resposta sexual saudável } \\
\text { (desejo, excitação, } \\
\text { orgasmo e resolução). }\end{array}$ & $\begin{array}{l}\text { Fatores relacionados: } \\
\text { medo; ansiedade, } \\
\text { distúrbio de autoimagem; } \\
\text { diminuição da lubrificação } \\
\text { vaginal; estenose vaginal; } \\
\text { sangramento vaginal } \\
\text { anormal. } \\
\text { Condições associadas: } \\
\text { radioterapia; } \\
\text { quimioterapia. } \\
\text { População em risco: } \\
\text { mulheres com idade igual } \\
\text { ou maior a } 40 \text { anos. }\end{array}$ & $\begin{array}{c}\text { Características } \\
\text { definidoras: abandono da } \\
\text { companheira; insatisfação } \\
\text { sexual; alteração do } \\
\text { orgasmo; dispareunia. }\end{array}$ \\
\hline $\begin{array}{l}\text { Qualitative } \\
\text { accounts } \\
\text { of patients' } \\
\text { determinants of } \\
\text { vaginal dilator } \\
\text { use after pelvic } \\
\text { radiotherapy }{ }^{25}\end{array}$ & 2015 & & $\begin{array}{l}\text { Fatores relacionados: } \\
\text { diminuição da lubrificação } \\
\text { vaginal; diminuição da } \\
\text { libido; estenose vaginal; } \\
\text { sangramento vaginal } \\
\text { anormal. } \\
\text { Condições associadas: } \\
\text { radioterapia. }\end{array}$ & $\begin{array}{c}\text { Características } \\
\text { definidoras: insatisfação } \\
\text { sexual; alteração do } \\
\text { orgasmo; dispareunia. }\end{array}$ \\
\hline $\begin{array}{c}\text { Quality of life } \\
\text { (QOL) in women } \\
\text { treated for } \\
\text { gynecologic } \\
\text { malignancies with } \\
\text { radiation therapy: } \\
\text { a literature review } \\
\text { of patient-reported } \\
\text { outcomes }^{26}\end{array}$ & 2014 & & $\begin{array}{l}\text { Fatores relacionados: } \\
\text { ansiedade; distúrbio de } \\
\text { autoimagem; diminuição } \\
\text { da lubrificação vaginal. } \\
\text { Condições associadas: } \\
\text { radioterapia. }\end{array}$ & $\begin{array}{c}\text { Características } \\
\text { definidoras: indiferença } \\
\text { do parceiro; dispareunia. }\end{array}$ \\
\hline
\end{tabular}

Fonte: Elaborada pelos autores, Recife, PE, Brasil, 2020 
Quadro 1. Continuação...

\begin{tabular}{|c|c|c|c|c|}
\hline Título & Ano & Atributos & Antecedentes & Consequentes \\
\hline $\begin{array}{l}\text { An examination } \\
\text { of the sexual } \\
\text { functions of } \\
\text { patients who } \\
\text { underwent a } \\
\text { gynecologic } \\
\text { cancer operation } \\
\text { and received } \\
\text { brachytherapy }\end{array}$ & 2018 & $\begin{array}{l}\text { Alteração na estrutura } \\
\text { do epitélio vaginal; } \\
\text { diminuição dos níveis de } \\
\text { estradiol e progesterona. }\end{array}$ & $\begin{array}{l}\text { Fatores relacionados: } \\
\text { comunicação deficiente } \\
\text { entre profissional- } \\
\text { paciente; vergonha; } \\
\text { distúrbio de autoimagem; } \\
\text { diminuição da libido; } \\
\text { estenose vaginal. } \\
\text { Condições associadas: } \\
\text { radioterapia; } \\
\text { quimioterapia; cirurgia. }\end{array}$ & $\begin{array}{c}\text { Características } \\
\text { definidoras: insatisfação } \\
\text { sexual; alteração do } \\
\text { orgasmo; dispareunia. }\end{array}$ \\
\hline $\begin{array}{l}\text { Impact of cervical } \\
\text { cancer on quality } \\
\text { of life: beyond the } \\
\text { short term (Results } \\
\text { from a single } \\
\text { institution) })^{28}\end{array}$ & 2015 & & $\begin{array}{l}\text { Fatores relacionados: } \\
\text { medo; distúrbio de } \\
\text { autoimagem; diminuição } \\
\text { da libido; diminuição da } \\
\text { lubrificação vaginal. } \\
\text { Condições associadas: } \\
\text { cirurgia. }\end{array}$ & $\begin{array}{c}\text { Características } \\
\text { definidoras: insatisfação } \\
\text { sexual; alteração do } \\
\text { orgasmo. }\end{array}$ \\
\hline $\begin{array}{l}\text { Sexuality of } \\
\text { Moroccan } \\
\text { Survivors of } \\
\text { Cervical Cancer: a } \\
\text { prospective data }{ }^{29}\end{array}$ & 2018 & & $\begin{array}{l}\text { Fatores relacionados: } \\
\text { comunicação deficiente } \\
\text { entre profissional- } \\
\text { paciente; atividades } \\
\text { de educação em saúde } \\
\text { deficiente; ansiedade; } \\
\text { diminuição da libido. } \\
\text { Condições associadas: } \\
\text { radioterapia; cirurgia; } \\
\text { depressão. }\end{array}$ & $\begin{array}{c}\text { Características } \\
\text { definidoras: insatisfação } \\
\text { sexual; dispareunia. }\end{array}$ \\
\hline
\end{tabular}

Fonte: Elaborada pelos autores, Recife, PE, Brasil, 2020

após o tratamento, algumas mulheres apresentam incontinência urinária e intestinal, o que acarreta sentimentos negativos em relação ao próprio corpo, como vergonha e medo. O medo da perda involuntária da urina durante $o$ ato sexual e a vergonha pela falta de controle do próprio corpo fazem com que o sexo ocorra de forma menos espontânea, levando à disfunção sexual ${ }^{35}$.

Estudo realizado em Amsterdam - cujo objetivo era avaliar as experiências sexuais e as necessidades de apoio psicossexual de pacientes e seus parceiros após a conclusão do tratamento para o câncer de colo do útero -, identificou que mais da metade das participantes da pesquisa apresentaram sensação de ansiedade em relação à dor durante a penetração vaginal, independentemente de essa experiência ter sido vivenciada anteriormente. Foi possível observar que muitos parceiros se sentiam angustiados devido ao medo de ferir sua companheira durante $o$ ato sexual ${ }^{35}$.

Sentimentos de culpa, perda e aflição, em relação à sexualidade vivenciada após o tratamento, permitiram aos pesquisadores chegarem à conclusão de que essas pacientes se encontravam sexualmente angustiados e experimentavam algum grau de disfunção sexual ${ }^{35}$.
O distúrbio de autoimagem leva a mulher à disfunção sexual, pois a percepção que uma pessoa tem de si própria influencia como ela irá desenvolver suas relações pessoais, e, dentro desse contexto, a sexualidade será expressa por meio de desejos, pensamentos, fantasias, valores, comportamentos e papéis sociais. Nesse sentido, as alterações corporais causadas pelo tratamento radioterápico influenciam negativamente no modo como a mulher enxerga sua feminilidade e seu corpo, o que causa diminuição tanto da autoestima quanto do desejo sexual ${ }^{1,36}$.

Outro fator relacionado à disfunção sexual identificado no estudo foi a ausência de parceiro fixo. Para alguns autores, a presença de um parceiro fixo reduz as chances de disfunção sexual feminina devido à segurança que o relacionamento proporciona à mulher, pois ela tem mais intimidade com seu corpo e de seu parceiro, consegue quebrar tabus morais, e desempenha sua sexualidade de forma satisfatória ${ }^{34,37,38}$.

Dessarte, ao estudar a presença de problemas sexuais, ansiedade e depressão em mulheres jovens e correlacionar seus achados com o status atual do relacionamento das participantes, pesquisadores brasileiros observaram que as mulheres solteiras apresentaram prevalência significativamente maior de problemas 
nos domínios lubrificação ( $45,3 \%)$, orgasmo $(53,1 \%)$, satisfação $(67,2 \%)$ e dor $(50 \%)$ - e também nos escores totais do Índice de Função Sexual Feminina (60,9\%) - em comparação com o grupo de mulheres que se encontravam em um relacionamento estável, corroborando com os resultados deste estudo, apesar de a população estudada não ter sido submetida à radioterapia para tratamento de câncer do colo do útero ${ }^{39}$.

A disfunção sexual caracteriza-se pela alteração em uma das fases da resposta sexual saudável, sendo elas: desejo, excitação, orgasmo e resolução 16,31,37,40,41. Entretanto, questões sociais, culturais e emocionais também precisam ser vistas e investigadas, para que a sexualidade feminina seja compreendida em sua totalidade.

É comum o relato de diminuição do desejo sexual por mulheres tratadas das neoplasias do colo do útero, além do sentimento de perda da feminilidade. Alterações na estrutura vaginal, decorrentes do tratamento, levam à diminuição da libido devido à ansiedade e ao medo de sentir dor durante o ato sexual, inclusive antes que a penetração vaginal ocorra ${ }^{35}$.

A diminuição da lubrificação vaginal também é uma queixa comum de mulheres tratadas do câncer do colo do útero e contribui para o desenvolvimento da disfunção sexual devido ao desconforto provocado durante a penetração vaginal. A menor lubrificação gera atrito na parede vaginal, que se encontra atrofiada, e o resultado é dor durante o intercurso sexual, sendo necessária a utilização de hidratantes vaginais para redução desses sintomas ${ }^{42}$. O edema local ocasionado pela obstrução linfática provoca sentimentos de constrangimento na mulher pela aparência de seu órgão genital, levando à diminuição da autoestima e menor desejo sexual ${ }^{22,37}$.

O tratamento radioterápico provoca a estenose e a diminuição do comprimento vaginal, cujas alterações estruturais causam um impacto negativo na sexualidade feminina em virtude da diminuição da sensibilidade do clitóris e da mucosa vaginal, além de reduzir a extensão da penetração vaginal; consequentemente, a mulher irá apresentar menor satisfação sexual ${ }^{43}$.

A alteração presente na estrutura vaginal após o tratamento radioterápico leva ao sangramento excessivo durante o coito, em decorrência da inflamação local, a qual, muitas vezes, está acompanhada de edema, perda da elasticidade e ulceração do tecido vaginal. A presença de sangramento durante o intercurso sexual causa desconforto e constrangimento à mulher, dificultando o relaxamento da musculatura pélvica e provoca sentimentos negativos quanto ao ato sexual ${ }^{5}$.

Diminuição da elasticidade vaginal, diminuição do comprimento vaginal, atrofia de parede, fibrose pélvica e dispareunia ocasionadas pelo tratamento radioterápico também são fatores contribuintes para o desenvolvimento da disfunção sexual feminina, conforme observado em estudo realizado na Suécia, com mulheres sobreviventes de câncer de colo do útero que haviam sido submetidas a tratamento radioterápico ${ }^{44}$.

Essas alterações causam intenso sofrimento, tanto de ordem física quanto psicológica; assim, muitas mulheres não se sentem mais atraentes, e o medo de abandono do parceiro é constante, além da dor experimentada durante a relação sexual, decorrente das alterações estruturais do epitélio vaginal ${ }^{35}$.

Dentre as condições associadas à disfunção sexual identificadas na população estudada, têm-se cirurgia, radioterapia e quimioterapia. Os efeitos secundários desses tratamentos causam diminuição da lubrificação vaginal, edema local e alterações importantes na estrutura do epitélio vaginal, levando à morbidade vaginal22.

O tratamento cirúrgico pode ter como consequência o encurtamento vaginal, diminuição da lubrificação vaginal, linfedema e alterações na bexiga e intestino, o que acarretará dispareunia e dificuldades para se atingir o orgasmo. A radioterapia, por sua vez, causa cicatrizes nos tecidos irradiados, provocando a estenose progressiva da vagina, impedindo completamente a penetração, sendo necessárias ações educativas em saúde que orientem as pacientes quanto às medidas preventivas ${ }^{45}$.

O tratamento quimioterápico, por sua vez, ocasiona perda de cabelo, fadiga, alterações na pele, diminuição de peso, náuseas e diarreia, suscitando o desenvolvimento de distúrbios de autoimagem e de identidade sexual, além de quadros depressivos ${ }^{45}$. Assim, as mulheres passam a desenvolver uma imagem errônea de si próprias, muitas não se sentem mais atraentes para os seus parceiros, e o medo da rejeição encontra-se presente. Tais sentimentos diminuem o desejo sexual, gerando a disfunção sexual feminina ${ }^{46}$. Estudo ${ }^{41}$ realizado no Departamento de Ginecologia e Obstetrícia de um hospital de nível terciário na Índia, entre mulheres diagnosticadas com quadro de depressão maior, observou que $90 \%$ das participantes da pesquisa possuíam algum grau de disfunção sexual, corroborando os achados desta pesquisa, a qual identificou a depressão como fator associado à disfunção sexual.

Quanto ao nível de escolaridade, a literatura assinala que mulheres com menos de oito anos de estudo têm menor compreensão acerca de suas questões sexuais, assim como menor acesso à informação, fato que gera barreiras ao desenvolvimento pleno de sua sexualidade ${ }^{36}$. Em um estudo realizado no Peru, com o objetivo de identificar os fatores associados à disfunção sexual de mulheres atendidas em um Hospital Universitário de nível terciário, os pesquisadores concluíram que o menor nível de escolaridade estava associado à presença de disfunção sexual na população estudada ${ }^{31}$.

Outra condição associada identificada para a disfunção sexual, nesse grupo de mulheres, foi o fato de estarem com uma ocupação laboral informal. Ter uma ocupação formal permite à mulher experimentar a sensação de maior segurança em relação a si e suas necessidades de saúde, o que a torna mais independente e reflete, de forma positiva, em sua sexualidade ${ }^{47}$.

Quanto à população em risco, percebeu-se que ter idade igual ou maior que 40 anos classificou essa população no grupo de risco para o desenvolvimento da disfunção sexual feminina, conforme apontado na literatura ${ }^{31,40}$. Nessa idade, há uma redução de $25 \%$ das fantasias sexuais, além de alterações fisiológicas e estruturais do organismo - como atrofia vaginal e alterações hormonais -, as quais contribuem para menor desejo sexual ${ }^{46}$. 
Três estudos ${ }^{24,25,27}$ identificaram a indiferença do parceiro e o abandono como consequentes da disfunção sexual feminina, além do sentimento de obrigatoriedade para a relação sexual. Esses dados corroboram estudo realizado no Peru, cujo objetivo foi identificar os fatores associados à disfunção sexual em mulheres atendidas em uma unidade de saúde de nível terciário. Os resultados da pesquisa apontaram as seguintes causas para disfunção sexual feminina: conflito conjugal, abuso emocional e ter relação sexual de forma indesejada ou por obrigação ${ }^{31}$.

Para obter um bom desempenho sexual, a mulher precisa ser sexualmente responsiva, receber amor, afeto e sentir-se pertencente a alguém. Desse modo, ao ser tratada com indiferença pelo parceiro ou sofrer abandono, ela desencadeará sentimentos negativos com relação a si própria e ao seu corpo, e ter dificuldades para se relacionar sexualmente devido a bloqueios mentais, o que poderá levar à disfunção sexual ${ }^{48}$.

Muitas mulheres relatam dificuldade em falar abertamente sobre sua sexualidade com seu parceiro, ocasionando distanciamento na relação. E mesmo com dificuldades para sentir prazer, elas acabam tendo relação sexual para satisfazer seus parceiros, esse sentimento de obrigatoriedade gera diminuição da libido e, consequentemente, a disfunção sexual ${ }^{35}$.

Essa disfunção pode ser representada pela diminuição da satisfação sexual, assim como pela diminuição da frequência das relações sexuais. A excitação subjetiva feminina é mais consistente que a modulação da vasocongestão genital; ou seja, a satisfação sexual feminina acontece sem necessariamente a mulher ter chegado à fase de orgasmo. Para tanto, ela precisa estar conectada de forma positiva com seu parceiro ${ }^{49}$.

Distúrbios de excitação, lubrificação, orgasmo, satisfação e dor são domínios que afetam a sexualidade feminina e provocam a disfunção sexual, dentre os quais se destacam os distúrbios de orgasmo e desejo ${ }^{30,37,38,47}$. Esses distúrbios associam-se à comunicação deficiente com o parceiro, ausência de preliminares e religião. A baixa frequência das relações sexuais, o desemprego, menopausa e a presença de uma doença subjacente também são considerados fatores de risco para a disfunção sexual ${ }^{30}$.

Estudo realizado na região Sul do Brasil, com o objetivo de estudar a função sexual e a qualidade de vida de mulheres que sobreviveram ao câncer ginecológico, entre os anos de 2009 e 2013 , observou que mais de $40 \%$ das mulheres participantes tornaram-se inativas sexualmente após o tratamento, o que lhes confere menor qualidade de vida ${ }^{36}$.

As mulheres que se encontravam sexualmente ativas após o tratamento para o câncer ginecológico, geralmente apresentavam disfunção sexual, dispareunia e estenose vaginal ${ }^{36}$. A dispareunia, quando presente, causa na mulher o medo de sentir dor durante o intercurso sexual, o que gera uma maior tensão na musculatura pélvica e consequentemente a penetração vaginal torna-se mais difícil, acarretando sentimentos negativos para o ato sexual ${ }^{50}$.

Nesse contexto, as ações de educação em saúde promovem o resgate do autoconhecimento e da confiança em si mesmas, além de exercerem influência positiva na saúde emocional dessas mulheres, uma vez que a recuperação da autoconfiança gera maior sensação de controle sobre o próprio corpo, e maior relaxamento da musculatura pélvica durante o intercurso sexual ${ }^{51}$.

\section{CONCLUSÃO E IMPLICAÇÕES PARA A PRÁTICA}

Os dados do estudo evidenciaram que a alteração na estrutura do epitélio vaginal, a diminuição dos níveis de estradiol e progesterona, a influência de fatores psicológicos, sociais e culturais, e a alteração em uma das fases da resposta sexual saudável constituem os principais atributos críticos do conceito de disfunção sexual em mulheres com câncer de colo do útero, submetidas a tratamento radioterápico.

Também foi possível observar que alterações na estrutura corporal, déficit de conhecimento, comunicação deficiente, sentimentos negativos, condição socioeconômica, idade e tratamento realizado são os principais antecedentes da disfunção sexual feminina. Da mesma forma, diminuição da frequência das relações sexuais, diminuição da satisfação sexual, diminuição do orgasmo, dispareunia, indiferença do parceiro, abandono e sentimento de obrigatoriedade caracterizam os consequentes do conceito. Por isso, a partir dos componentes identificados no estudo, foi possível compreender com mais clareza o conceito estudado, fornecendo elementos que subsidiem a prática clínica do enfermeiro, no que diz respeito ao planejamento de ações de educação em saúde e prevenção de distúrbios na sexualidade feminina.

Os achados deste estudo reiteram a necessidade de mais pesquisas sobre a disfunção sexual feminina-em especial para população de mulheres com diagnóstico de câncer do colo do útero que são submetidas a tratamento radioterápico -, sobretudo no campo da enfermagem, pois o enfermeiro exerce papel fundamental na realização de ações de promoção, prevenção e reabilitação em saúde.

O número reduzido de estudos disponíveis na literatura $e$ o baixo nível de evidência dos estudos encontrados referentes ao tema, na área de enfermagem, constituíram as principais limitações desta revisão.

\section{CONTRIBUIÇÕES DOS AUTORES}

Desenho do estudo. Thaynan Gonçalves da Silva. Sheila Coelho Ramalho Vasconcelos Morais. Francisca Márcia Pereira Linhares.

Aquisição de dados. Thaynan Gonçalves da Silva. Francisca Márcia Pereira Linhares.

Análise de dados. Thaynan Gonçalves da Silva. Kilders Mitshel Lucas de Oliveira. Sheila Coelho Ramalho Vasconcelos Morais. Jaqueline Galdino Albuquerque Perrelli. Santana de Maria Alves de Sousa. Francisca Márcia Pereira Linhares.

Interpretação dos resultados. Thaynan Gonçalves da Silva. Kilders Mitshel Lucas de Oliveira. Sheila Coelho Ramalho Vasconcelos Morais. Jaqueline Galdino Albuquerque Perrelli. Santana de Maria Alves de Sousa. Francisca Márcia Pereira Linhares. 
Redação e revisão crítica do manuscrito. Thaynan Gonçalves da Silva. Kilders Mitshel Lucas de Oliveira. Sheila Coelho Ramalho Vasconcelos Morais. Jaqueline Galdino Albuquerque Perrelli. Santana de Maria Alves de Sousa. Francisca Márcia Pereira Linhares.

Aprovação da versão final do artigo. Thaynan Gonçalves da Silva. Kilders Mitshel Lucas de Oliveira. Sheila Coelho Ramalho Vasconcelos Morais. Jaqueline Galdino Albuquerque Perrelli. Santana de Maria Alves de Sousa. Francisca Márcia Pereira Linhares.

Responsabilidade por todos os aspectos do conteúdo e a integridade do artigo publicado. Thaynan Gonçalves da Silva. Kilders Mitshel Lucas de Oliveira. Sheila Coelho Ramalho Vasconcelos Morais. Jaqueline Galdino Albuquerque Perrelli.Santana de Maria Alves de Sousa. Francisca Márcia Pereira Linhares.

\section{EDITOR ASSOCIADO}

Stela Maris de Mello Padoin (1)

\section{EDITOR CIENTÍFICO}

Ivone Evangelista Cabral (1)

\section{REFERÊNCIAS}

1. Fakunle IE, Maree JE. Sexual function in South African women treated for cervical cancer. Int. J. Africa Nurs. Sci. 2019;10:124-30. http://dx. doi. org/10.1016/j.jians.2019.04.002.

2. Instituto Nacional de Câncer José Alencar Gomes da Silva. Coordenação de Prevenção e Vigilância. Estimativa 2020: incidência de câncer no Brasil. Rio de Janeiro: INCA; 2019. 120 p.

3. Ribeiro CM, Silva GA. Avaliação da produção de procedimentos da linha de cuidado do câncer do colo do útero no Sistema Único de Saúde do Brasil em 2015. Epidemiol Serv Saude. 2018;27(1):e20172124. http:// dx.doi.org/10.5123/S1679-49742018000100004.

4. Figueiredo E, Monteiro M, Ferreira A. Tratado de oncologia. São Paulo: Revinter; 2013.2300 p.

5. Aydin R, Oskay YU. Sexual experience of women after pelvic radiotherapy for cervical cancer. Turk. J. Oncol. 2016;31(4):119-27.

6. Grion RC, Baccaro LF, Vaz AF, Costa-Paiva L, Conde DM, Pinto-Neto AM. Sexual function and quality of life in women with cervical câncer before radiotherapy: a pilot study. Arch Gynecol Obstet. 2016;293(4):879-86. http://dx.doi.org/10.1007/s00404-015-3874-z. PMid:26319157.

7. Denlinger CS, Carlson RW, Are M, Baker KS, Davis E, Edge SB et al. Survivorship: sexual dysfunction (Female), Version 1.2013. J Natl Compr Canc Netw. 2014;12(2):184-92. http://dx.doi.org/10.6004/ jnccn.2014.0019.

8. Musa J. The need for societal investment to improve cervical cancer outcomes in Nigeria: a commentary. Afr J Reprod Health. 2017;21(4):1723. http://dx.doi.org/10.29063/ajrh2017/v2114.2. PMid:29624947.

9. Hammerschmidt KSA, Rosa LM, Alvarez AM, Radunz V, Tomasi AVR, Valcarenghi RV. Comportamento sexual das mulheres em tratamento radioterápico. Ciênc Cuid Saúde. 2016 jan/mar;15(1):194-201. http:// dx.doi.org/10.4025/cienccuidsaude.v15i1.25064.

10. Foerster R, Schnetzke L, Bruckner T, Arians N, Rief H, Debus J et al. Prognostic factors for long-term quality of life after adjuvant radiotherapy in women with endometrial cancer. Strahlenther Onkol. 2016;192(12):895904. http://dx.doi.org/10.1007/s00066-016-1037-1. PMid:27605238.

11. Levin AO, Carpenter KM, Fowler JM, Brothers BM, Andersen BL, Maxwell GL. Sexual morbidity associated with poorer psychological adjustment among gynecological cancer survivors. Int J Gynecol Cancer. 2010 abr;20(3):461-70. http://dx.doi.org/10.1111/IGC.0b013e3181d24ce0. PMid:20375814.

12. Walker LO, Avant KC. Strategies for theory construction in nursing. 5th ed. Upper Saddle River: Pearson/Prentice Hall; 2011.

13. Rodgers BL, Knafl KA. Concept development in nursing: foundations, techniques and applications. Philadelphia: W.B. Saunders; 2000.

14. Whittemore R, Knafl $\mathrm{K}$. The integrative review: updated methodology. J Adv Nurs. 2005;52(5):546-53. http://dx.doi.org/10.1111/j.13652648.2005.03621.x. PMid:16268861.

15. The Joanna Briggs Institute - JBI. Joanna Briggs Institute Reviewers Manual: 2014 edition [Internet]. South Australia: The Joanna Briggs Institute/The University of Adelaide; 2014 [citado 2020 jan 14]. Disponível em: https://nursing.Isuhsc.edu/JBI/docs/ReviewersManuals/Economic. pdf

16. NANDA Internacional. Diagnósticos de enfermagem da NANDA-I

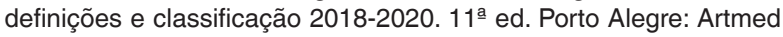
2018.

17. Mangueira SO. Revisão do diagnóstico de enfermagem Processos familiares disfuncionais relacionados a abuso de álcool [tese]. Fortaleza (CE): Departamento de Enfermagem, Universidade Federal do Ceará 2014.

18. Critical Appraisal Skills Programme - CASP. Critical appraisal checklists [Internet]. Oxford: CASP; 2018 [citado 2020 jan 14]. Disponível em: https://casp-uk.net/casp-tools-checklists/

19. Melnyk BM, Fineout-Overholt E. Evidence-based practice in nursing \& healthcare: a guide to best practice. Philadelphia: Lippincott Williams \& Wilkins; 2010

20. Ferrari AAC. Solução salina versus solução de heparina na perviedade do cateter venoso periférico na terapia venosa intermitente: revisão sistemática [dissertação]. Ribeirão Preto (SP): Escola de Enfermagem de Ribeirão Preto, Universidade de São Paulo; 2016.

21. Samaie Nouroozi A, Alyasin A, Malek Mohammadi A, Mehrdad N, Mousav $\mathrm{SA}$, Vaezi M et al. Autologous platelet-released growth fator and sexua dysfunction amendment: a pilot clinical trial of successful improvement sexual dysfunction after pelvic irradiation. Asian Pac J Cancer Prev. 2019;20(3):817-23. http://dx.doi.org/10.31557/APJCP.2019.20.3.817. PMid:30911301.

22. Hofsjö A, Bergmark K, Blomgren B, Jahren $\mathrm{H}$, Bohm-Starke $\mathrm{N}$ Radiotherapy for cervical cancer - impact on the vaginal epithelium and sexual function. Acta Oncol. 2017a;57(3):338-45. http://dx.doi. org/10.1080/0284186X.2017.1400684. PMid:29140150.

23. Zhou W, Yang X, Dai Y, Wu Q, He G, Yin G. Survey of cervical cancer survivors regarding quality of life and sexual function. J Cancer Res Ther. 2016;12(2):938-44. http://dx.doi.org/10.4103/0973-1482.175427. PMid:27461678.

24. Jensen PT, Froeding LPF. Pelvic radiotherapy and sexual function in women. Transl Androl Urol. 2015;4(2):186-205. PMid:26816824.

25. Bakker RM, Vermeer WM, Creutzberg CL, Mens JW, Nout RA, Ter Kuile MM. Qualitative accounts of patients'determinants of vaginal dilatador use after pelvic radiotherapy. J Sex Med. 2015;12(3):764-73. http:// dx.doi.org/10.1111/jsm.12776.

26. Mirabeau-Beale KL, Viswanathan NA. Quality of life (QOL) in women treated for gynecologic malignancies with radiation therapy: a literature review of patient-reported outcomes. J Sex Med. 2015;12:764-73.

27. Guner O, Gumussoy S, Celik N, Saruhan A, Kavlak O. An examination of the sexual functions of patientes who underwent a gynecologic cancer operation and received brachytherapy. Pak J Med Sci. 2018;34(1):15-9. http://dx.doi.org/10.12669/pjms.341.14241. PMid:29643871.

28. Khalii J, Bellefqih S, Sahli N, Afif M, Elkacemi H, Elmajjaoui S et al. Impact of cervical cancer on quality of life: beyond the short term (Results from a single institution): quality of life in long-term cervical cancer survivors: results from a single institution. Gynecol Oncol Res Pract. 2015 set 19;2(1):7. http://dx.doi.org/10.1186/s40661-015-0011-4. PMid:27231567.

29. Dahbi Z, Sbai A, Mezouar L. Sexuality of morroccan survivors of cervical cancer: a prospective data. Asian Pac J Cancer Prev. 2018;19(11):3077-9. http://dx.doi.org/10.31557/APJCP.2018.19.11.3077. PMid:30485944. 
30. Espitia De La Hoz FJ. Prevalencia y caracterización de las disfunciones sexuales en mujeres, en 12 ciudades colombianas, 2009-2016. Rev Colomb Obstet Ginecol. 2018 mar 28;69(1):9. http://dx.doi.org/10.18597/ rcog.3035.

31. Diaz-Velez C, Samalvides-Cuba F. Factores asociados a disfunción sexual en mujeres atendidas en un hospital nivel III-Chiclayo-Perú. Rev Chil Obstet Ginecol. 2017;82(4):386-95. http://dx.doi.org/10.4067/ s0717-752620170004003686.

32. World Health Organization - WHO. Defining sexual health: report of a technical consultation on sexual health 28-31 January 2002, Geneva [Internet]. Geneva: WHO; 2002 [citado 2020 jan 14]. Disponível em: https://www.who.int/reproductivehealth/publications/sexual_health/ defining_sexual_health.pdf?ua=1

33. McCabe MP, Sharlip ID, Atalla E, Balon R, Fisher AD, Laumann E et al. Definitions of sexual dysfunctions in women and men: a consensus statement from the fourth international consultation on sexual medicine 2015. J Sex Med. 2016;13(2):135-43. http://dx.doi.org/10.1016/j. jsxm.2015.12.019. PMid:26953828.

34. Almeida MJS, Peixoto C, Ribeiro TTSB, Silva LM, Veras AB. Terapia cognitivo-comportamental em grupo para a disfunção sexual na pós-menopausa. J Bras Psiquiatr. 2018;68(4):231-8. http://dx.doi. org/10.1590/0047-2085000000210.

35. Vermeer WM, Bakker RM, Kenter GG, Stiggelbout AM, Ter Kuile MM Cervical cancer survivors' and partners' experiences with sexual dysfunction and psychosexual support. Support Care Cancer. 2016;24(4):1679-87. http://dx.doi.org/10.1007/s00520-015-2925-0. PMid:26412245.

36. Nascimento FC, Deitos J, Luz CM. Comparação da disfunção do assoalho pélvico com função sexual e qualidade de vida em sobreviventes ao câncer ginecológico. Cad. Bras. Ter. Ocup. 2019;27(3):628-37. http:// dx.doi.org/10.4322/2526-8910.ctoAO1640.

37. Bezerra KC, Feitoza SR, Vasconcelos CTM, Karbage SAL, Saboia DM, Oriá MOB. Função sexual de universitárias: estudo comparativo entre Brasil e Itália. Rev Bras Enferm. 2018;71(suppl 3):1511-7.

38. Satake JT, Pereira TRC, Aveiro MC. Self-reported assessment of female sexual function among Brazilian undergraduate healthcare students: a cross-sectional study (survey). Sao Paulo Med J. 2018;136(4):333-8. http://dx.doi.org/10.1590/1516-3180.2018.0005240418. PMid:30110073.

39. Pereira VM, Nardi AE, Silva AC. Sexual dysfunction, depression, and anxiety in young women according to relationship status: an online survey. Trends Psychiatry Psychother. 2013;35(1):55-61. http://dx.doi. org/10.1590/S2237-60892013000100007. PMid:25923186.

40. Dall'Agno ML, Ferreira CF, Ferreira FV, Pérez-López FR, Wender MCO. Validação do índice de funcionamento sexual feminino-6 em mulheres brasileiras de meia-idade. Rev Bras Ginecol Obstet. 2019;41(7):432-9. PMid:31344717.
41. Sreelakshmy K, Velayudhan R, Kuriakose D, Nair R. Sexual dysfunction in females with depression: a crosssectional study. Trends Psychiatry Psychother. 2017;39(2):106-9. http://dx.doi.org/10.1590/2237-60892016-0072. PMid:28700039.

42. Boa R, Grénman S. Psychosexual health in Gynecologic Cancer. Int J Gynaecol Obstet. 2018;143(suppl. 2):147-52. http://dx.doi.org/10.1002/ ijgo.12623. PMid:30306581.

43. Oskay ÜY, Beji NK, Bal MD, Yilmaz SD. Evoluation of sexual function in patients with gynecologic cancer and evidence-basead nursing interventions. Sex Disabil. 2011;29(1):33-41. http://dx.doi.org/10.1007/ s11195-010-9188-y.

44. Hofsjö A, Bohm-Starke N, Blomgren B, Jahren H, Steineck G, Bergmark K. Radiotherapy-induced vaginal fibrosis in cervical câncer survivors. Acta Oncol.2017b;56(5):661-6. http://dx.doi.org/10.1080/0284186X.2016.1275778. PMid:28084859.

45. Graziottin A, Serafini A, Palacios S. Aetiology, diagnostic algorithms and prognosis off female sexual dysfunction. Maturitas. 2019;63(2):128-34. http://dx.doi.org/10.1016/j.maturitas.2009.04.007. PMid:19493639.

46. Salomão PB, Navarro PA, Romão APMS, Lerri MR, Lara LAS. Função sexual de mulheres com infertilidade. Rev Bras Ginecol Obstet. 2018;40(12):771-8. PMid:30381810.

47. Guendler JA, Katz L, Flamini MEDM, Lemos A, Amorim MM. Prevalência de disfunções sexuais e seus fatores associados em gestantes de uma clínica de pré-natal. Rev Bras Ginecol Obstet. 2019;41(9):555-63. PMid:31480080.

48. Primo WQSP, Corrêa FJS, Brasileiro JPB. Manual de Ginecologia da Sociedade de Ginecologia e Obstetrícia de Brasília. $2^{\underline{a}}$ ed. Brasília: Editora Luan Comunicação; 2017.

49. Basson R, Brotto LA, Laan E, Redmond G, Utian WH. Assessment and management of women's sexual dysfunctions: problematic desire and arousal. J Sex Med. 2005;2(3):291-300. http://dx.doi.org/10.1111/j.17436109.2005.20346.x. PMid:16422860.

50. Huffman LB, Hartenbach EM, Carter J, Rash JK, Kushner DM Maintaining sexual health throughout gynecologic cancer survivorship: a comprehensive review and clinical guide. Gynecol Oncol. 2016;140(2):359-68. http://dx.doi.org/10.1016/j.ygyno.2015.11.010. PMid:26556768.

51. Lee Y. Patients' perception and adherence to vaginal dilator therapy: a systematic review and synthesis employing symbolic interactionism. Patient Prefer Adherence. 2018;12:551-60. http://dx.doi.org/10.2147/ PPA.S163273. PMid:29695897. 
No artigo "Disfunção sexual em mulheres com câncer do colo do útero submetidas à radioterapia:análise de conceito", DOI número: https://doi.org/10.1590/2177-9465-EAN-2020-0404, publicado em Escola Anna Nery Revista de Enfermagem 2021;25(4):e20200404,

onde se lê: "Jaqueline Galdino Albuquerque Perreli”;

leia-se: “Jaqueline Galdino Albuquerque Perrelli”. 